\title{
Outbreak of extended spectrum $\beta$ lactamase producing Klebsiella pneumoniae in a neonatal unit
}

\author{
Jennifer Royle, Sharon Halasz, Gillian Eagles, Gwendolyn Gilbert, Dianne Dalton, \\ Peter Jelfs, David Isaacs
}

\begin{abstract}
An outbreak of extended spectrum $\beta$ lactamase producing Klebsiella pneumoniae (ESBLKp) in a neonatal unit was controlled using simple measures. Normally, the control of such infections can be time consuming and expensive. Seven cases of septicaemia resulted in two deaths. ESBLKp isolates were subtyped by pulsed field gel electrophoresis, and four of the five isolates typed were identical. Control of the outbreak was achieved by altered empiric antibiotic treatment for late onset sepsis and prevention of cross infection by strict attention to hand washing. Widespread colonisation of babies in the unit was presumed, so initial surveillance cultures were not performed. No further episodes of sepsis occurred. (Arch Dis Child Fetal Neonatal Ed 1999;80:F64-F68)
\end{abstract}

Keywords: $\beta$ lactamase producing Klebsiella pneumoniae; antibiotic resistance; hygiene; sepsis

Gram negative bacillary sepsis is a common nosocomial problem in neonatal units. Australian data from 1992-3 revealed coagulase negative staphylococcus as the pathogen in about half the late onset cases of sepsis and that Gram negative bacteria were responsible for around $20 \%$ of late onset infections. ${ }^{1}$

Outbreaks of Klebsiella infections in neonatal units have been widely reported and are frequently associated with widespread colonisation of babies, systemic infections, and death. ${ }^{2-5}$ Reports of recent outbreaks highlight the problem of mulitresistant strains of Klebsiella. ${ }^{6-9}$ The presence of Klebsiella in high numbers in the stool provides a reservoir for spread from baby to baby, with transfer on the hands of staff, and, indeed, positive culture results from staff members' hands have been obtained. $^{81011}$ Colonised babies are the commonest reported source of infection, although many primary environmental reservoirs of Gram negative bacteria have been reported in epidemics, including distilled water containers, ${ }^{12}$ resuscitation apparatus, ${ }^{13}{ }^{14}$ hand washing brushes ${ }^{15}$ and bottles of $1 \%$ chlorxylenol soap. ${ }^{16}$

Methods reported to control outbreaks have included reducing implementing "antibiotic pressure" by changing the antibiotic policy, introducing methods to improve hand washing, cohorting (nursing together) infected and colonised babies, and varying levels of surveillance cultures to detect environmental con- tamination. These methods can prove very expensive.

We describe a first stage approach to an outbreak of a multiresistant extended spectrum $\beta$ lactamase producing Klebsiella pneumoniae (ESBLKp), which involves education of staff to improve hand washing and changes to the antibiotic policy. No initial surveillance cultures of babies or environment were done. Since the epidemic resolved after these simple measures had been taken, further more costly methods were not required.

\section{Methods}

The neonatal unit at The Royal Alexandra Hospital for Children is a tertiary referral centre. All babies are referred from outside hospitals, there being no attached obstetric unit. There are 27 beds: 10 intensive care, 12 extended care, and five isolation beds in two isolation rooms. Twelve members of the nursing team are on duty at any one time, one, two or three of whom are senior staff. Three neonatologists work one week on at a time. Day shifts are staffed by one medical registrar and two residents. Overnight cover is provided by one medical registrar. The unit moved in October 1995 from an old hospital site in Camperdown to a new site in Westmead.

Routine surveillance cultures of babies are not performed in the unit. In general, babies over 48 hours old with suspected late onset sepsis are treated empirically with vancomycin and gentamicin. A third generation cephalosporin (cefotaxime) is used in place of gentamicin when Gram negative sepsis is strongly suspected.

Pulsed field gel electrophoresis (PFGE) was performed on available isolates. Agarose plugs containing the isolates were each digested overnight using the restriction endonucleases Xbal and Nsi1. PFGE was performed using a Biorad Chef-Mapper. The digested plugs were incorporated into a $1 \%$ agarose gel which was electrophoresed using the following conditions: a 21 hour run time, temperature of $14^{\circ} \mathrm{C}$, voltage of 6.0 volts $/ \mathrm{cm}$ and switch times of 2 seconds to 40 seconds linear ramping.

The babies' characteristics are shown in table 1. On 30 July 1995, a full term baby with bladder extrophy (case 1) became clinically septic. She was known from superficial wound cultures to be colonised with an ESBLKp. Empiric treatment was started using flucloxacillin and amikacin. Blood and urine cultures subsequently grew ESBLKp that was sensitive to amikacin. The baby recovered uneventfully.

The second case occurred three months later, when a term baby who had been treated 
for necrotising enterocolitis, and not known to be colonised with ESBLKp, developed septicaemia. Treatment with cefotaxime and gentamicin was started, as Gram negative sepsis was suspected. After ESBLKp had been isolated amikacin was substituted and the baby recovered.

Cases 3 and 4 occurred after a further 10 weeks, and cases 5 and 6 another five weeks later. Two babies died; in each case empirical antibiotic treatment was begun to which the ESBLKp subsequently proved resistant. Case 7 occurred one week after cases 5 and 6 , by which time the empiric antibiotic choice had been changed in view of the outbreak. The organisms in cases 1 and 2 were sensitive to amikacin and imipenem. Cases 3 to 6 grew organisms resistant to amikacin and sensitive only to imipenem.

One other unit baby developed endotracheal tube colonisation with the ESBLKp strain on 2 March 1996. This baby died on 4 March from causes other than sepsis. In March 1996 an urgent meeting was held with the infection control team and the neonatologists to decide strategies to prevent further cases, and to decide how the unit antibiotic policy should be changed.

\section{INTERVENTION}

The infectious diseases team and neonatologists decided on strategies to deal with the outbreak. The empirical antibiotic policy for suspected late onset sepsis was changed to imipenem and vancomycin. Surveillance cultures of babies or of the environment were not performed immediately, as negative cultures would not reliably exclude colonisation or contamination. The primary source of infection was presumed to be colonised infants, with gut colonisation as the major reservoir.

All babies in the unit at the time of the outbreak were presumed to be colonised. Case 6 was placed in one of the two isolation rooms, as she was known to be colonised. All babies were to be regarded as potential reservoirs for further spread. New (presumably uncolonised) babies admitted to the unit were nursed in areas within the two main rooms of the nursery with the colonised babies.

The primary focus for preventing cross contamination was hand washing. In-service edu- cation sessions explaining the outbreak to the nursing staff and unit nursing educators were conducted. The importance of hand washing to prevent baby to baby spread of gut colonising organisms was stressed. The wearing of disposable gloves for nappy changing, a policy previously only variably practised, was reinforced.

Nurses were declared to be the advocates for the babies. Each nurse was given the responsibility to act on behalf of the babies under his or her care, and to insist that all attending personnel wash hands before and after handling the baby. Any medical or paramedical staff refusing to wash his or her hands after being asked to do so by the attending sister was to be reported to the infection control team. A high level of motivation emerged among the nursing staff: "nurse power" advocacy was well received by visiting staff.

Attendance of at least one of the infectious disease team at a weekly neonatal ward round was routine. However, on each occasion during the subsequent 10 weeks, the outbreak was discussed and the importance of hand washing reiterated.

\section{Results}

No further episodes of sepsis occurred after case 7 . Ten weeks after the antibiotic policy was changed, fecal or rectal swab cultures from all the babies in the unit were taken to assess the point prevalence of colonisation.

Fecal culture from case 6 was positive for ESBLKp but only one other baby, admitted two weeks earlier, was positive. This baby was placed in the isolation room with case 6 . Apart from case 6 , all babies on the unit had been admitted after the epidemic intervention had started. Repeat surveillance cultures performed one week later on all babies on the unit confirmed positive cultures only in the same two babies in the isolation room.

The empirical antibiotic policy for suspected late onset sepsis was changed back to vancomycin and gentamicin for all unit babies, other than for the two babies in isolation who were to be treated empirically with vancomycin and imipenem if they became clinically septic.

Subtyping of the ESBLKp isolates was performed on cases 3-7. The isolates from the earlier two cases were not available for testing.

Table 1 Cases of ESBLKp sepsis

\begin{tabular}{|c|c|c|c|c|c|c|c|c|c|c|}
\hline Cases & Sex & $\begin{array}{l}\text { Gestation } \\
\text { (weeks) }\end{array}$ & $\begin{array}{l}\text { Birthweight } \\
\text { (kg) }\end{array}$ & Underlying problems & $\begin{array}{l}\text { Antibiotics before } \\
\text { ESBLKp sespis } \\
\text { and days of use }\end{array}$ & $\begin{array}{l}\text { Age at } \\
\text { sepsis onset } \\
\text { (days) }\end{array}$ & $\begin{array}{l}\text { Date of } \\
\text { ESBLK } \\
\text { sepsis }\end{array}$ & $\begin{array}{l}\text { Sites of } \\
\text { positive } \\
\text { cultures }\end{array}$ & Empiric antibiotics & Outcome \\
\hline 1 & $\mathrm{~F}$ & 41 & 3.5 & Bladder extrophy & $\begin{array}{l}\text { A2, G2, } \\
\text { Am(oral) } 5\end{array}$ & 24 & $30 / 7 / 95$ & $\begin{array}{l}\text { Blood, } \\
\text { urine }\end{array}$ & Ami, F & Survived \\
\hline 2 & M & 40 & 3.7 & NEC (day 3) & A9. G9, M9 & 13 & $\begin{array}{l}29 / 10 / \\
95\end{array}$ & Blood & Ctx, G then Ami (at 24 hours) & Survived \\
\hline 3 & M & 34 & 2.0 & $\begin{array}{l}\text { Malrotation; volvulus; NEC } \\
\text { (day 25) }\end{array}$ & $\begin{array}{l}\mathrm{Cl} 12, \mathrm{G} 14, \mathrm{~A} 7, \\
\mathrm{M} 4\end{array}$ & 60 & $19 / 1 / 96$ & Blood & Ctx, $\mathrm{G}$ then Imi (at 24 hours) & Survived \\
\hline 4 & $\mathrm{~F}$ & 35 & 2.4 & Gastoschisis & $\begin{array}{l}\text { A11, Ami11, } \\
\text { M5, G5 }\end{array}$ & 56 & $27 / 1 / 96$ & Blood & $\begin{array}{l}\text { V, G then Ctx, G (at } 24 \text { hours) } \\
\text { then Imi (1 dose) }\end{array}$ & Died \\
\hline 5 & M & 29 & 1.2 & HMD & $\mathrm{Nil}$ & 6 & $3 / 3 / 96$ & Blood & $\mathrm{V}, \mathrm{G}$ & Died \\
\hline 6 & $\mathrm{~F}$ & 25 & 0.6 & $\begin{array}{l}\text { CLD, FTT; NEC, multiple } \\
\text { gut resections }\end{array}$ & $\begin{array}{l}\text { Multiple } \\
\text { courses }\end{array}$ & 195 & $3 / 3 / 96$ & Blood & A, $G$ then Imi (at 48 hours) & Survived \\
\hline 7 & $\mathrm{~F}$ & 37 & 2.82 & $\begin{array}{l}\text { Cloacal extrophy; } \\
\text { lipomyelo-meningocele }\end{array}$ & $\mathrm{A} 6, \mathrm{G} 6, \mathrm{M} 4$ & 6 & $11 / 3 / 96$ & $\begin{array}{l}\text { Blood, } \\
\text { urine }\end{array}$ & Imi & Survived \\
\hline
\end{tabular}

$\mathrm{HMD}=$ hyaline membrane disease CLD = chronic lung disease FTT = failure to thrive NEC = necrotising enterocolitis; ESBLKp = extended spectrum $\beta$-lactamase producing Klebsiella pneumoniae; $\mathrm{A}=$ ampicillin; $\mathrm{Cl}=$ clindamycin; $\mathrm{Imi}=$ imipenem; $\mathrm{Am}=$ amoxycillin; $\mathrm{Ctx}=\mathrm{cefotaxime} ; \mathrm{M}=\operatorname{metronidazole} ; \mathrm{Ami}=$ amikacin; $\mathrm{F}=$ flucloxacillin; $\mathrm{V}=$ vanomycin; $\mathrm{G}=$ gentamicin. 


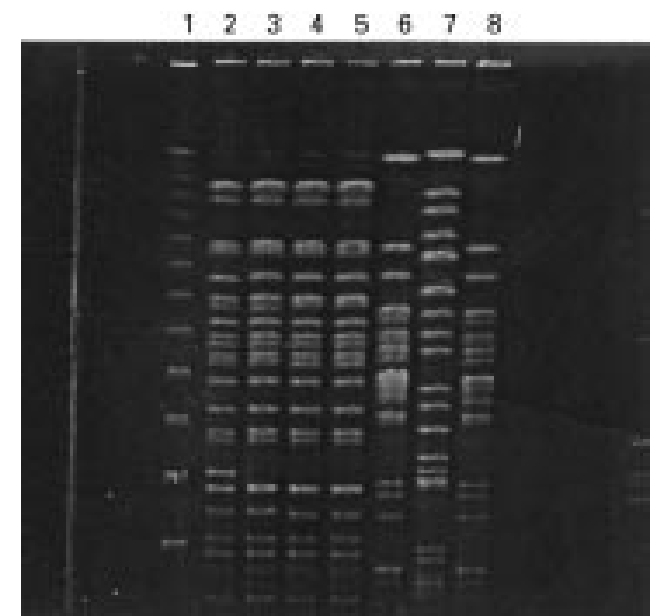

Figure 1 Pulse field gel electrophoresis: Lane 1 Lamda marker; lanes 2 to $5 \mathrm{~K}$ pneumoniae isolates from cases 3 to 6; lane 6 isolate from case 7; lane 7 ESBL negative $K$ pneumoniae control; lane 8 ESBL positive K pneumoniae control.

With each restriction endonuclease enzyme, identical banding patterns were displayed by isolates from cases 3-6, with case 7 having a distinctly different pattern (fig 1).

\section{Discussion}

Hospital neonates develop gastrointestinal tract colonisation with Klebsiella, Enterobacter, and Citrobacter species (KEC species) at high rates compared with well babies at home in whom Escherichia coli is the predominant bowel flora. The risk of stool colonisation with KEC species is increased with over three days of antibiotic use and with duration of hospital stay, $60 \%$ being colonised by day 15 and over $90 \%$ by day $30 .{ }^{17}$

Microbial drug resistance is an inescapable consequence of the widespread use of antimicrobial agents. Outbreaks of infection with resistant strains of Gram negative organisms create the situation where empirical antibiotic choice may not cover the causative organism. Resistance to aminoglycosides has been the focus of many reported outbreaks. ${ }^{6}{ }^{18}{ }^{19}$ Amikacin resistant Enterobacteriaceae were reported from Louisville in 1980; colonised babies were the only source discovered and there were three deaths. ${ }^{20}$ Extended spectrum $\beta$ lactamase (ESBL) producing strains of Gram negative bacilli were first reported in Germany in 1983. These organisms showed decreased susceptibility to the third generation cephalosporins, due to a plasmid mediated $\beta$ lactamase identified as a mutational modification of the Klebsiella SHV-1 enzyme, designated Klebsiella SHV-2. ${ }^{21}$ More than 27 distinct enzymes have been described, with worldwide distribution. The selective pressure of heavy cephalosporin use has probably contributed to the emergence of ESBL producing organisms. ${ }^{22-24}$ Outbreaks are frequent as host strains are readily transferred..$^{892526}$

A nursery epidemic can be defined as a significant increase in the rate of certain infections over baseline. ${ }^{27}$ At our hospital, all episodes of blood culture confirmed sepsis in the nursery have been documented prospectively since
1991, so an increase in the incidence of Klebsiella sepsis was not difficult to document. The death of case 5, presumably as a result of cross contamination, caused the infection control team to intervene. In retrospect, earlier intervention-for example, when cases 3 and 4 presented-would have been preferable and might have averted the later cases.

Subtyping revealed that a single epidemic organism was responsible for cases 3-6. The isolates from cases 1 and 2 were not available for subtyping, while case 7 was caused by a different strain of ESBLKp of unknown origin. The origin of the predominant ESBLKp is unknown, although we suspect that it was introduced by the transfer of a colonised baby from another hospital. We looked at the antibiotics received by our colonised and infected babies, and none of them had received any third generation cephalosporins, nor prolonged courses of other antibiotics. However, as we do not perform surveillance cultures on admission to the unit, we are unable to say whether the first baby was already colonised before admission.

The ESBLKp isolate from case 7 occurring at the height of the epidemic was not a genetic match with the other isolates. This highlights the benefits of subtyping organisms in an epidemic and raises further questions relating to the origins of different strains of ESBLKp.

Surgical patients were over represented in our outbreak - six of the seven patients having undergone recent surgical procedures. Because our unit is tertiary referral centre, several surgical patients are on the unit at any one time. Many non-surgical patients were colonised or presumed to be colonised, and only one became septic. This suggests that surgery may be a contributory factor. Gastrointestinal surgery, and in particular, ischaemic bowel, may be an important risk factor for sepsis. Other outbreaks have also reported over representation of surgical patients. ${ }^{88}$ An outbreak of ESBLKp at Guy's Hospital, London, was mostly seen in neonates recovering from cardiac surgery for congenital defects. ${ }^{8}$ The increased risk of clinical infection is probably associated with invasive procedures in general, and not restricted to major surgery. This highlights the relative likelihood of such an epidemic becoming a rapid clinical problem in a major surgical neonatal centre compared with smaller units where surgery is not performed on site.

Various outbreak management strategies have been described. The methods used address antibiotic policy, cohorting, hand washing, wearing of gloves and gowns, and environmental swabs. The expense of the management increases as the number of methods instituted rises. In some outbreaks short term antibiotic policy change has been reported as being adequate to prevent further spread of infection. ${ }^{29}{ }^{30}$ Occasionally, an environmental source has been found and removal of the environmental source has led to rapid resolution of the outbreak. ${ }^{12}{ }^{15}$.

Many outbreaks are managed with surveillance cultures and cohorting. If this approach is 
taken, regular, preferably weekly, cultures need to be performed until the outbreak is resolved, to ensure accurate cohorting. The lower cost approach is to assume that all babies are colonised and that strict attention to hand washing is promoted. New admissions are then nursed with babies presumed to be colonised, but are protected by improved hand washing routines.

The outbreak in our unit was managed by this low cost approach. It was felt that immediate surveillance cultures would not significantly change our approach to the outbreak and would add unnecessary cost. Klebsiella outbreaks are consistently associated with high levels of colonisation. ${ }^{245}$ Regardless of the level of colonisation we could not isolate large numbers of babies in the unit and would still have relied on increased hand washing to eradicate spread. Case 6 was isolated because persistent colonisation was assumed after confirmed infection, and because the increased awareness and discussion of the implications of multiresistant organisms, associated with nursing her in isolation, was felt to be advantageous.

In most Klebsiella epidemics, the primary source of infection is gut colonisation of infants. $^{245}$ We began stage 1 management without searching for an environmental source and, as no further episodes of sepsis occurred, it is likely that none existed. If further cases had occurred we would have begun a second stage of management and surveyed the unit environment. One may question the possible continued risk to babies by not seeking out a possible external source, but with the change in antibiotic policy the risk to infants was reduced.

There is little published evidence to support the control of outbreaks by improved hand washing and the eventual discharge of colonised babies from the unit. Compliance with hand washing by staff has rarely been observed to occur on more than one third of occasions of patient contact, ranging from $28 \%-48 \% .^{31-34}$ Tibballs at the Royal Children's Hospital (RCH) in Melbourne recently reported baseline hand washing rates by Intensive Care Unit medical staff of only $12.4 \%$ before and $10.6 \%$ after patient contact, and only $4.3 \%$ of occasions both before and after patient contact. ${ }^{35}$ The $\mathrm{RCH}$ rates increased to about 1 in 3 occasions when the doctors knew they were being observed. However, with education, feedback reinforcement, and further observations the rates rose to $68.3 \%$ before, $64.8 \%$ after, and $55.2 \%$ both before and after patient contact. Rates after the study period ended were still well above 1 in 3, although they had fallen. ${ }^{35}$ How to maintain high long term rates of hand washing is unknown, but Tibballs' study confirms that short term hand washing improvement is possible. If this can be initiated at the time of an outbreak, it may prevent further cross contamination as the incentive to prevent further deaths in an epidemic is probably the best motivation for staff. However, most hand washing studies are not performed during epidemics. ${ }^{32}$ We did not formally measure whether our educational approach to improved hand washing affected compliance. The rapid resolution of the outbreak may have been coincidental or due to altering the antibiotic policy alone.

The methods for improving hand washing practices and their success are varied. Our approach to set up the nurse caring for each baby on a particular shift as the advocate has not been described before. For several weeks after the outbreak, no nurse would let anyone touch the baby under his or her care without hand washing before and after contact, and by inference the nurses were practising better hand washing skills themselves. This advocacy should be encouraged on a long term basis.

We thank the staff of the Grace neonatal unit at the Royal Alexandra Hospital for Children and the neonatologists Peter Barr, Robert Halliday and Julian Wojtulewicz

1 Isaacs D, Barfield C, Clothier T, et al. Late-onset infections of infants in neonatal units. $F$ Paediatr Child Health 1996;32:158-61.

2 Hable KA, Matsen JM, Wheeler DJ, et al. Klebsiella type 33 septicemia in an infant intensive care unit. $f$ Pediatr 1972;80:920-4.

3 Kayyali MZ, Nicholson DP, Smith IM. A Klebsiella outbreak in a pediatric nursery: emergency action and
preventative surveillance. Clin Pediatr 1972;11:422-6.

preventative surveillance. Clin Pediatr 1972;11:422-6.
4 Hill HR, Hunt CE, Matson JM. Nosocomial colonization with Klebsiella, type 26 , in a neonatal intensive care unit associated with an outbreak of sepsis, meningitis and necrotizing enterocolitis. F Pediatr 1974;85:415-9.

5 Eisenach KD, Reber RM, Eitzman DV, et al. Nosocomial infections due to kanamycin-resistant, (R)-factor carrying enteric organisms in an intensive care nursery. Pediatrics 1972;50:395-402

6 Shamseldin el Shafie S, Smith W, Donnelly G. An outbreak of gentamicin-resistant Klebsiella pnuemoniae in a neonatal ward. Cent Eur $\mathcal{F}$ Pub Health 1995;3:129-31.

7 Fidel-Rimon O, Leibovitz E, Juster-Reicher A, et al. An outbreak of multiresistant Klebsiella at the Neonatal Intensive Care Unit, Kaplan Hospital, Rehovot, Israel, November 1991-April 1992. Am f Perinatol 1996;13:99-102.

8 French GL Shannon KP, Simmons J. Hospital outbreak of Klebsiella pneumoniae resistant to broad-spectrum cephaosporins and beta-lactam-beta-lactamase inhibitor combinations by hyperproduction of SHV-5 beta-lactamase. $\mathcal{F}$ Clin Microbiol 1996;34:358-63.

9 Hobson RP, MacKenzie FM, Gould IM. An outbreak of multiply resistant Klebsiella pneumoniae in the Grampians region of Scotland. F Hosp Infect 1996;33:249-62.

10 Smith PJ, Brookfield DSK, Shaw DA, et al. An outbreak of Serratia marcescens infection in a neonatal unit. Lancet 1984;i:151-3.

11 Adams BG, Marrie TJ. Hand carriage of aerobic gramnegative rods may not be transient. F Hyg 1982;89:33-46.

12 Rapkin RH. Pseudomonas cepacia in an intensive care nursery. Pediatrics 1976;57:239-43.

13 Drewitt SE, Payne DJH, Tuke W, et al. Eradication of Pseudomonas aeruginosa infection from a special care nursery. Lancet 1972; i: $946-8$.

14 Stone JW, Das BC. Investigation of an outbreak of infection with Acinetobacter calcoaceticus in a special care baby unit. F Hosp Infect 1985;6:42-8.

15 Anagnostakis D, Fitsialos J, Koutsia C, et al. A nursery outbreak of Serratia marcescens infection. Evidence of a single source of contamination. Am $f$ Dis Child 1981;135:413-5.

16 Archibald L K, Corl A, Shah B, et al. Serrratia marcescens outbreak associated with extrinsic contamination of $1 \%$ chlorxylenol soap. Infect Control Hosp Epidemiol 1997; 18:704-9.

17 Goldmann DA, Leclair J, Macone A. Bacterial colonization of neonates admitted to an intensive care environment. $\mathcal{F}$ Pediatr 1978;93:288-93.

18 Oxley VM, Bird TJ, Grieble HG et al. Hospital isolates of Serratia marcescens transferring ampicillin, carbenicillin and gentamicin resistance to other Gram-negative bacteria including Pseudomonas aeruginosa. Antimicrob Agents Chemother 1979;15:93-100.

19 Franco JA, Eitzman DV, Baer H, et al. Antibiotic usage and microbial resistance in an intensive care nursery. Am f D is Child 1973;126:318-21.

20 Cook LN, Davis RS, Stover BH. Outbreak of amikacinresistant enterobacteriaceae in an intensive care nursery. Pediatrics 1980;65:264-8.

21 Payne DJ, Amyer SB. Transferable resistance to extended spectrum $\beta$-lactamase: A major threat or minor inconvenience? F Antimicrob Chemother 1990;27:255-61.

22 Modi N, Damjanovic V, Cooke RW. Outbreak of cephalosporin resistant Enterobacter cloacae infection in a neonatal intensive care unit. Arch Dis Child 1987;62:148-51. 
23 Jacoby GA. Antimicrobial resistant pathogens in the 1990s. Ann Rev Med 1996;47:169-79.

24 Pena $C$, Pujol M, Ardanuy $C$, et al. Epidemiology and successful control of a large outbreak due to Klebsiella pneumoniae producing extended spectrum $\beta$ lactamases: Are we losing the battle? Aust f Med Sci 1995;16: 16-25

25 Tristam S. Extended spectrum $\beta$-lactamases : Are we losing the battle? Antimicrob Agents Chemother 1998;42:53-8.

26 Martinez-Martinez L, pascual A, Jacoby GA. Quinolone resistance from a transferable plasmid. Lancet 1998;i: 797-9.

27 Peter G, Cashore WJ. Infections acquired in the nursery: epidemiology and control. In: Remington JS, Klein JO. Infectious diseases of the fetus and newborn infant. 4th edn. Philadelphia, W B Saunders, 1995:1275.

28 Quinn J P. Clinical significance of extended spectrum $\beta$-lactamases. Eur $\mathcal{F}$ Clin Microbiol Infect Dis 1994;13:39-42.
29 Franco JA, Eitzman DV, Baer H, et al. Antibiotic usage and microbial resistance in an intensive care nursery. Am $\mathcal{F}$ Dis microbial resistance in a

30 Raz R, Sharir R, Shilowitz L, et al. The elimination of gentamicin-resistant Gram negative bacteria in a newborn intensive care unit. Infection 1987;15:32-4.

31 Pritchard RC, Raper RF. Doctors and handwashing: instilling Semmelweis' message. Med F Aust 1996;164:389-90.

32 Albert RK, Condie F. Hand-washing patterns in medical intensive-care units. N Engl F Med 1981;304:1465-6.

33 Doebbeling BN, Stanley GL, Sheetz CT, et al. Comparative efficacy of alternative hand-washing agents in reducing nosocomial infections in intensive care units. $N$ EngI $\mathcal{F}$ Med 1992;327:88-93.

34 Goldmann D, Larson E. Handwashing and nosocomial infections. N EngI f Med 1992; 327:120-2.

35 Tibballs J. Teaching hospital medical staff to handwash. Med 7 Aust 1996; 164: 395-8. 